\title{
Use of Prolate Spheroidal Wave Functions in the Time Domain First Order Reliability Method
}

Jensen, Jørgen Juncher

Published in:

Practical Design of Ships and Other Floating Structures. PRADS 2019.

Link to article, DOI:

10.1007/978-981-15-4624-2_46

Publication date:

2021

Document Version

Peer reviewed version

Link back to DTU Orbit

Citation (APA):

Jensen, J. J. (2021). Use of Prolate Spheroidal Wave Functions in the Time Domain First Order Reliability Method. In T. Okada, Y. Kawamura, \& K. Suzuki (Eds.), Practical Design of Ships and Other Floating Structures. PRADS 2019. (pp. 760-769). Springer. Lecture Notes in Civil Engineering Vol. 63 https://doi.org/10.1007/978981-15-4624-2_46

\section{General rights}

Copyright and moral rights for the publications made accessible in the public portal are retained by the authors and/or other copyright owners and it is a condition of accessing publications that users recognise and abide by the legal requirements associated with these rights.

- Users may download and print one copy of any publication from the public portal for the purpose of private study or research.

- You may not further distribute the material or use it for any profit-making activity or commercial gain

- You may freely distribute the URL identifying the publication in the public portal 


\title{
Use of Prolate Spheroidal Wave Functions in the Time Domain First Order Reliability Method
}

\author{
Jørgen Juncher Jensen \\ Dept. of Mechanical Engineering, Technical University of Denmark, Kgs. Lyngby, Denmark
}

jjj@mek.dtu.dk

\begin{abstract}
The paper consider the use of prolate spheroidal wave functions as basic orthogonal functions in the wave description in the time domain first order reliability method applied to wave response statistics in stationary sea states. Intact stability of a ship in beam sea is considered as an example and the results for the most probable response and the associated statistics are evaluated and compared to the usual procedure using trigonometric functions
\end{abstract}

Keywords: Critical wave episode, intact stability of ships, Wave functions

\section{Introduction}

The First Order Reliability Method (FORM) has proved to be an efficient method for extreme value predictions of various non-linear wave load responses of marine structures, e.g. Jensen (2009), Jensen (2015), Choi et al. (2017). In the time domain analyses the wave elevation and associated wave kinematics are discretized in a large number of trigonometric components, typical 30 to 100, each associated with a statistical independent random variable. Thereby, the FORM can be rather time-consuming due to the inherent optimization problem involving the same number of unknown. This is usually not a serious problem for simpler response models, e.g. 1D problems, but for $6 \mathrm{D}$ problems the calculation time might be very large, Choi et al. (2017). In order to reduce the number of statistical variables an interesting proposal is suggested by Sclavounos (2012), where the trigonometric basic functions in the wave load predictions are replaced by basic functions derived from prolate spheroidal wave functions (PSWF). Thereby, it might be expected that much fewer functions are needed to represent typical extreme wave load responses. For instance, an accurate estimation of the autocorrelation of the waves only requires 3 to 6 basic functions derived from the PSWF and the wave spectral density.

In the present study, the PSWF are generated using sampling theory, Khare and George (2003), which provides a very efficient procedure. The transformation from the PSWF to the trigonometric wave components needed in the wave response model does not require FFT as the Fourier components directly are the PSWF functions at the associated wave frequencies. Thus, it is easy to modify the standard FORM procedure based on trigonometric wave components to use PSWF as input. 
However, the application of PSWF to the time-domain FORM procedure might have some problems related especially to the memory effects of the wave response, because the time range covered by the PSWF is equal to the Slepian frequency $c$ defining the PSWF divided by the highest frequency in the wave spectrum. For typical wave spectra, this frequency is close to $1 \mathrm{rad} / \mathrm{sec}$, implying a time range close to $C$. Furthermore, the number of important PSWF components is approximately $2 c / \pi$ and this number should be much less than the trigonometric wave components typical used in FORM for wave response prediction. In the paper the application of PSWF to FORM is investigated using intact stability of a ship in beam sea as example.

\section{Prolate Spheriodal Wave Functions (PSWF)}

In the present study the PSWF are generated using the procedure described in Khare and George (2003). It uses the Whittaker-Shannon sampling theorem to determine the PSWF functions $\psi_{n}(x, c)$ :

$$
\psi_{n}(x, c)=\sum_{m=-\infty}^{\infty} \psi_{n}\left(\frac{\pi m}{c}, c\right) \frac{\sin (c x-\pi m)}{c x-\pi m}
$$

where $C$ is denoted the Slepian frequency, Sclavounos (2012). Eq. (1) represents an interpolation between known values of $\psi_{n}(x, c)$ at discrete values $x=\pi \mathrm{m} / c$. These values are determined by the algebraic eigenvalue problem, Khare and George (2003):

$$
\begin{aligned}
& \mu_{n} \psi_{n}\left(\frac{\pi m}{c}, c\right)=\frac{c}{\pi} \sum_{k=-\infty}^{\infty} B_{m k} \psi_{n}\left(\frac{\pi k}{c}, c\right) ; \quad-\infty<m<\infty \\
& B_{m k}=\int_{-1}^{1} \frac{\sin (c X-\pi m)}{c x-\pi m} \frac{\sin (c x-\pi k)}{c x-\pi k} d x
\end{aligned}
$$

The matrix $B_{m k}$ is real and symmetric and hence the eigenfunctions and eigenvalues are real. The eigenfunctions are ordered by decreasing eigenvalues starting from $n=0$. Furthermore, the matrix is also centrosymmetric implying that the eigenfunctions $\psi_{n}(x, c)$ are either even (for $n=0,2, .$. ) or odd (for $\left.n=1,3, ..\right)$ in $x$. In the numeric calculations the matrix is truncated to the dimension $N \times N$, i.e. the integers $m, k=-(N-1) / 2, . .,(N-1) / 2$. For $c=20, N=51$ proves sufficient. The matrix elements are calculated by a standard trapezoidal integration procedure. The largest eigenvalues are close to, but less than one. Only the largest $2 c / \pi$ eigenvalues are significant, after that they become close to zero. The eigenfunctions are normalized as

$$
\int_{-1}^{1} \psi_{m}(x, c) \psi_{n}(x, c) d x=\delta_{m n}
$$

where $\delta_{m n}$ is Kroneckers delta. For $|x|>1$ the eigenfunctions rapidly go to zero. 


\section{Wave Statistics Using PSWF}

Sclavounos (2012) suggests to use PSWF instead of trigonometric functions in the description of stationary stochastic waves in order to reduce the number of basic functions in time domain analyses. Here only the main results are quoted. For a derivation, the very readable treatment by Sclavounos (2012) should be consulted. In the present formulation, a one-sided wave spectrum $S(\omega)$ is assumed.

The time variation $t$ of the wave elevation $\varsigma(t)$ is written in the form:

$$
\begin{aligned}
& \varsigma(t)=\sum_{n=0}^{N_{\zeta}} z_{n} S_{n} f_{n}(t) ; \quad-T<t<T \\
& f_{n}(t)=\sum_{j=0}^{N_{e}} A_{n j} \psi_{j}\left(\frac{t}{T}, c\right)
\end{aligned}
$$

Here $Z_{n}$ are independent standard normal distributed variables. The number $N_{\varsigma}+1$ of components in the wave representation must be less than or equal to the number $N_{e}+1$ of PSWF used. The description is limited to the time range $-T<t<T$, where $T$ is the limit after which the PSWF rapidly decay to zero. This limit relates to the Slepian frequency $C$ and the upper limit $\Omega$ of the frequency range used for the wave spectrum by, Sclavounos (2012),

$$
c=T \Omega
$$

Hence, the higher the upper frequency limit $\Omega$ the smaller is the time range covered.

The coefficients (eigenvalues) $S_{n}$ and the matrix (eigenvectors) $A_{n j}$ follow from the solution of the algebraic eigenvalue problem:

$$
\begin{aligned}
& s_{n}^{2} A_{n i}=\lambda_{i} \sum_{j=0}^{N_{e}} \lambda_{j} G_{i j} A_{n j} ; \quad 0 \leq i \leq N_{e} \\
& G_{i j}=\Omega \int_{0}^{1} S(\omega=x \Omega) \psi_{i}(x, c) \psi_{j}(x, c) d x \text { if } i+j \text { even } \\
& G_{i j}=0 \text { if } i+j \text { odd }
\end{aligned}
$$

The coefficients $\lambda_{n}$ are related to the eigenvalues $\mu_{n}$ from Eq. (2) by

$$
\lambda_{n}= \pm \sqrt{\frac{2 \pi \mu_{n}}{c}}
$$

The sign in Eq. (7) is determined from relations 


$$
\begin{array}{ll}
2 \int_{0}^{1} \psi_{n}(u, c) \cos (c x u) d u=\lambda_{n} \psi_{n}(x, c) & \text { for even } n \\
2 \int_{0}^{1} \psi_{n}(u, c) \sin (c x u) d u=\lambda_{n} \psi_{n}(x, c) ; & \text { for odd } n
\end{array}
$$

Thus $\lambda_{n}<0$ for $n=2,6,10, \ldots$ and $n=3,7,11, \ldots$.

The summation in Eq. (4) is taken over PSWF with eigenvalues $\mu_{i}$ not close to zero, i.e. $N_{e} \approx 2 c / \pi$. The functions $f_{n}(t)$ are normalized such that

$$
\int_{-T}^{T} f_{m}(\tau) f_{n}(\tau) d \tau=T \delta_{m n}
$$

Due to the separation in Eq. (6) of contribution from even and odd PSWF terms, the functions $f_{n}(t)$ also become either even or odd in time $t$.

The wave description, Eq. (4), is clearly normal distributed with mean value zero due to $Z_{i}$ and as shown in Sclavounos (2012) allows a representation of the autocorrelation function $R(t)$ with only a small number of terms:

$$
R(t)=\int_{0}^{\Omega} S(\omega) \cos (\omega t) d \omega=\sum_{n=0}^{N_{\xi}} s_{n}^{2} f_{n}(t) f_{n}(0)
$$

This relation can serve as a check of the accuracy of the PSWF representation. Note that only the even functions of $f_{n}(t)$ enters Eq. (10) as the odd functions are zero at $t$ $=0$.

\section{$4 \quad$ First Order Reliability Method Using PSWF}

When using the First Order Reliability Method (FORM) for extreme value predictions of wave-induced loads, the wave elevation is usually given in terms of trigonometric components, e.g. Jensen (2009):

$$
\begin{aligned}
& \varsigma(t)=\sum_{i=1}^{M} \sigma_{i}\left(u_{i} \cos \omega_{i} t-\bar{u}_{i} \sin \omega_{i} t\right) \\
& \sigma_{i}=\sqrt{S\left(\omega_{i}\right) \Delta \omega}
\end{aligned}
$$


Here $u_{i}, \bar{u}_{i}$ are independent standard normal distributed variables and $\omega_{i}$ a set of discrete frequency covering the main part of the wave spectrum $S(\omega)$. Often an equidistant frequency spacing $\Delta \omega=\omega_{i}-\omega_{i-1}$ is chosen with $\Omega_{\text {lower }} \leq \omega_{i} \leq \Omega_{\text {upper }}=\Omega$. With the wave elevation given by Eq.(11) any non-linear wave response can be determined by a proper hydrodynamic model, see e.g. Jensen (2015) for examples.

In the FORM analysis, the design point $u_{i}, \bar{u}_{i}=u_{i}^{*}, \bar{u}_{i}^{*} ; i=1,2, . ., M$ corresponding to the most probable wave elevation leading to a given response is determined by a non-linear optimization procedure. As the number of unknowns $u_{i}, \bar{u}_{i}$ are $2 M$, it might require a very large computational effort for complicated non-linear hydrodynamic models, given that $M$ is typical of the order 15 to 50 . Therefore, if $u_{i}, \bar{u}_{i}$ are expressed in term of the variables $Z_{n}$ defining the stochastic properties in the PSWF wave elevation representation, Eq. (4), much fewer stochastic parameters $N_{\varsigma}$ have to be determined by the optimization procedure. The transformation between $Z_{n}$ and $u_{i}, \bar{u}_{i}$ are readily obtained due to the Fourier invariance of the PSWF, Eq.(8), without any needs for FFT analyses. Combining Eqs (4), (8) and (11) yields

$$
\begin{aligned}
& u_{i}=\frac{2}{\sigma_{i}} \frac{\Delta \omega}{\Omega} \sum_{n=0}^{N_{\varsigma}} z_{n} S_{n} \sum_{\substack{j=0 \\
j \text { even }}}^{N_{e}} \frac{A_{n j}}{\lambda_{j}} \psi_{j}\left(\frac{\omega_{i}}{\Omega}, c\right) \equiv \sum_{n=0}^{N_{\varsigma}} a_{i n} z_{n} \\
& \bar{u}_{i}=-\frac{2}{\sigma_{i}} \frac{\Delta \omega}{\Omega} \sum_{n=0}^{N_{\varsigma}} z_{n} S_{n} \sum_{\substack{j=1 \\
j \text { odd }}}^{N_{e}} \frac{A_{n j}}{\lambda_{j}} \psi_{j}\left(\frac{\omega_{i}}{\Omega}, C\right) \equiv \sum_{n=0}^{N_{\zeta}} b_{i n} z_{n}
\end{aligned}
$$

The deterministic coefficients $a_{i n}, b_{i n}$ in Eq. (12) are calculated before the FORM optimization. Thus, when the optimization routine has determined a new set of $N_{\varsigma}$ stochastic parameters $Z_{n}$, Eq.(12), provides the corresponding $2 M u_{i}, \bar{u}_{i}$ parameters for input to the hydrodynamic response model. The result is feed back to the optimization procedure and used to estimate a better estimate of the design point $z_{n}^{*}$ and thereby $u_{i}^{*}, \bar{u}_{i}^{*}$.

\section{Example: Intact Stability of a Ship in Beam Sea}

The use of PSWF in FORM is investigated by an example dealing with intact stability of a ship in beam sea. A 1D hydrodynamic model similar to the one used in Choi et al. (2017) is applied and the ship is the same, i.e. no forward speed and a GM=2.5m. However, only wave loads are included here, whereas the wind load is excluded for the sake 
of brevity.

A Pierson-Moskowitz wave spectrum with significant wave height $H_{S}=8 \mathrm{~m}$ and zero up-crossing period $T_{Z}=12 \mathrm{sec}$ is assumed. The cut-off frequencies are the same as in Choi et al. (2017):

$$
\Omega_{\text {lower }}=\frac{\pi}{T_{Z}} ; \quad \Omega_{\text {upper }}=\frac{3 \pi}{T_{Z}}=0.785 \mathrm{rad} / \mathrm{s}=\Omega
$$

In the FORM analysis in Choi et al. (2017) the number $M$ of frequency components in Eq.(11) was chosen to 30. It could, however, be reduced to 15 without changing the result. Thus if the present PSWF approach should be a good alternative in FORM applications, the number of PSWF functions should be much less than $2 M=30$, say, half the value. Hence, $c=20$ is a reasonable choice for the present example. From Eqs. (5), (14) the time range parameter in Eq. (4) becomes $T=25 \mathrm{sec}$, which is, however, rather short compared to a memory time of about $50 \mathrm{sec}$ for the present hydrodynamic model of the roll angle. If $c=40$ was chosen instead the memory time would have been covered better, but then the number of PSWF functions becomes close to the number of trigonometric terms in the existing wave description and the benefit of the PSWF vanishes. Hence, the present example will investigate how close the PSWF approach with $c=20$ can model the most probable wave and response scenario together with the associated statistics for a range of large roll angles.

In the calculation of the PSWF functions, Eq. (2), $N=51$ has been found to be sufficient for $c=20$. The eigenvalue problem, Eq.(6), are solved for $N_{e}=20$ and the eigenvalues $S_{n}$ are shown in Fig. 1. Eq. (4) shows that $S_{n}$ act as sort of wave amplitudes on each $f_{n}(t)$ because of the normalization, Eq. (9). Only the first 15 eigenvalues are different from zero, in agreement with $N_{\varsigma} \approx 2 c / \pi$. About half corresponds to even $f_{n}(t)$ functions, the rest to odd functions.

The autocorrelation $R(t)$ follows from Eq. (10) and is shown in Fig. 2. Clearly the sum of $s_{n}^{2} f_{n}(t) f_{n}(0)$ terms (green curve) is very close to the value obtained by direct integration of $S(\omega) \cos (\omega t)$ (red curve) within the range $0 \leq t \leq T=25 \mathrm{sec}$. However, outside this range the PSWF curve drops rapidly to zero and, hence, memory effects are not accounted for correctly for $t>25 \mathrm{sec}$. The figure also shows the contribution to $R(t)$ from the first four even $f_{n}(t)$ functions. The variance $R(0)$ is $3.75 \mathrm{~m}^{2}$, which differs slightly from the theoretical value $H_{S}^{2} / 16=4 \mathrm{~m}^{2}$ mostly due to the cutoff frequency $\Omega$. 


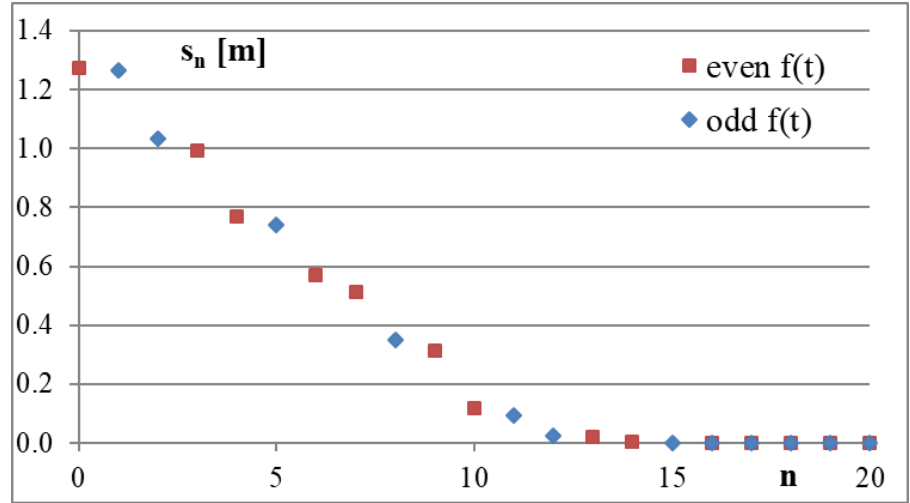

Fig. 1. Eigenvalues $S_{n}[\mathrm{~m}]$ from Eq. (6)

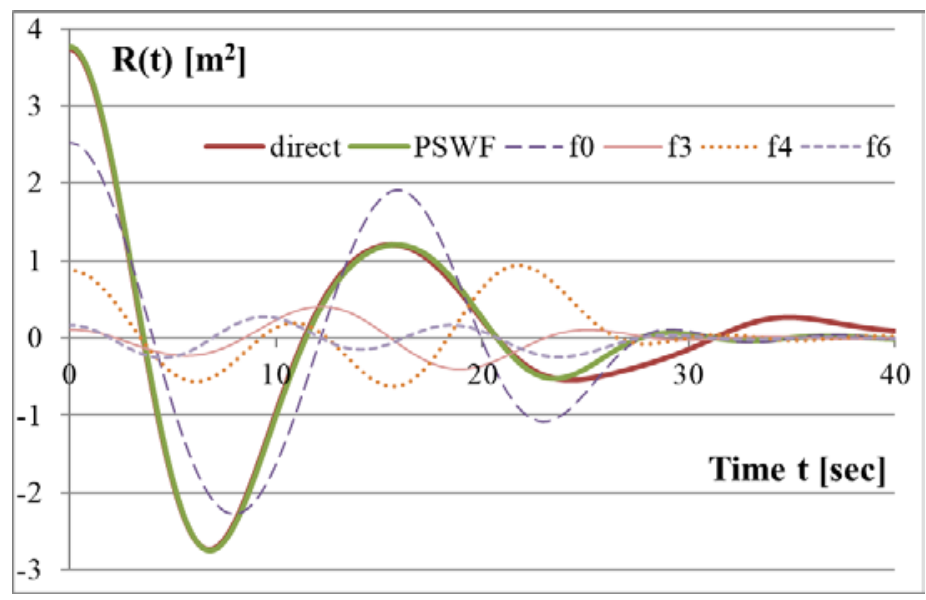

Fig. 2. Autocorrelation $R(t)$, Eq. (10). Direct integration (direct) and sum over

$$
s_{n}^{2} f_{n}(t) f_{n}(0) \text { terms (PSWF). }
$$

The findings above are in line with those in Sclavounos (2012) and show that within a short time range the use of PSWF can provide very accurate results with much fewer terms than by a trigonometric representation.

Implementation of the PSWF in the FORM procedure described in e.g. Choi et al. (2017) is straightforward using Eq. (12). The optimization for the design point is now performed in the variables $z_{i} ; i=1,2, . ., N_{\varsigma}$, where $N_{\varsigma}=13(\approx 2 c / \pi)$. This is a much smaller number than used in the original procedure with $2 M=60 u_{i}, \bar{u}_{i}$ variables. Therefore, the computational time is reduced by nearly an order of magnitude. The question is then how accurate the PSWF representation is regarding the extreme value statistics. 
The FORM procedure in Choi et al. (2017) using the trigonometric wave representation is executed with two set of input: A very accurate calculation using $2 M=100$ and a time $t_{0}=150 \mathrm{sec}$ between initialization of each time domain solution and the result (the roll angle) and a simpler one using $2 M=30$ and $t_{0}=75 \mathrm{sec}$. As initial values the roll angle and roll angle velocity are taken as zero at $t=-t_{0}$ and the target value is then at $t=0$. These two solutions are denoted "150s100trig" and "75s30trig" in Figs. 3-5. With the PSWF representation, a FORM solution is found with $2 M=100$ and $t_{0}=75 \mathrm{sec}$, denoted "75s100pswtrig". In this case, the number $M$ is nearly unimportant for the computational time as the optimization is done in the $N_{\varsigma}+1=14$ variables $z_{i}$ and with Eq. (12) applied for calculation of $u_{i}^{*}, \bar{u}_{i}^{*}$ using $z_{n}=z_{n}^{*}$. In all three cases the design point values $u_{i}^{*}, \bar{u}_{i}^{*}$ are used in the hydrodynamic calculations.

For a target roll angle of 34.4 deg ( 0.6 rad) the most probable wave elevation and corresponding roll angle response leading to this roll angle are shown in Fig. 3 and Fig. 4 , respectively.

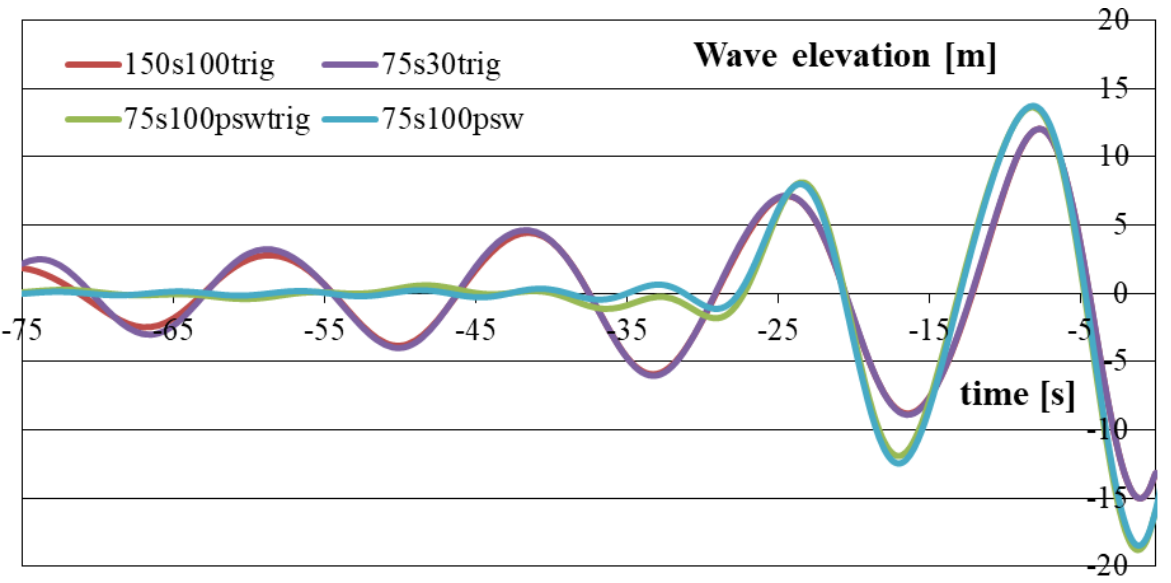

Fig. 3.Most probable wave scenario leading to a roll angle 34.4 deg at $\mathrm{t}=0$.

For the wave elevation in Fig. 3 an additional curve ("75s100psw”) based directly on Eq. (4) with $Z_{n}=Z_{n}^{*}$ is included, but as seen the two PSWF results "75s100pswtrig”, "75s100psw” are very close as they should be except for small numerical inaccuracies in the generation of the $f_{n}(t)$ functions. The same holds for the reliability index results in Fig.5. 


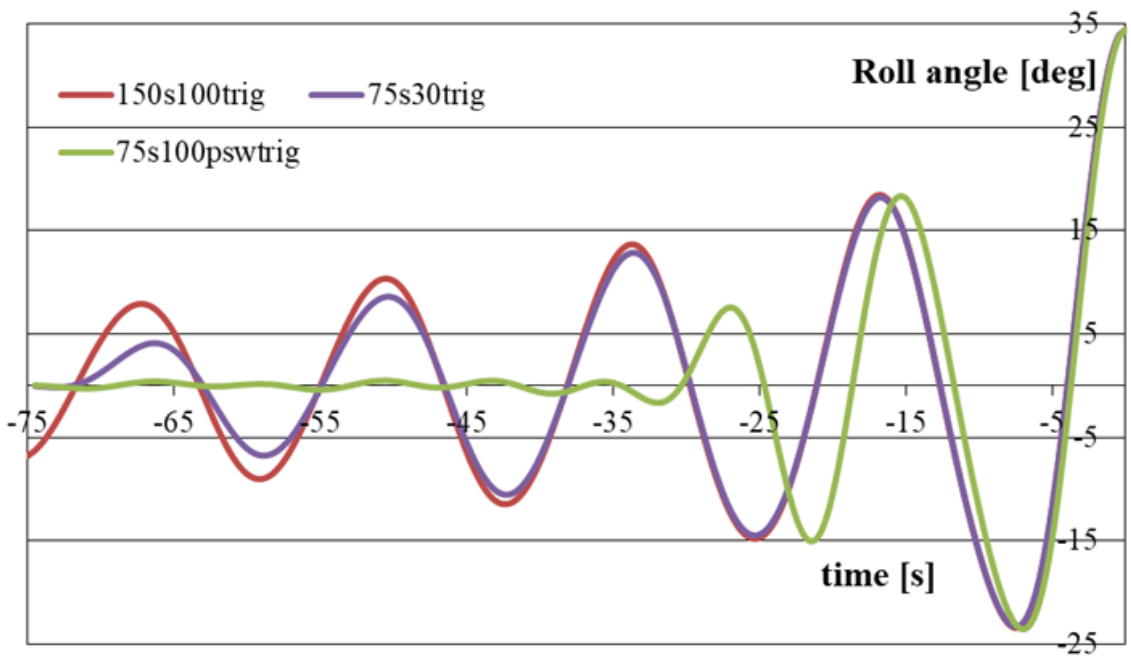

Fig. 4. Most probable roll angle scenario leading to a roll angle 34.4 deg at $\mathrm{t}=0$.

The two wave representations using $u_{i}, \bar{u}_{i}$ as optimization parameters yield very close results in Figs. 3, 4. However, the results using PSWF deviate significantly from these results. Especially, it is clear from Figs. 3, 4 that in the initiation period $-75 \mathrm{sec} \leq t<-25 \mathrm{sec}$ both a significant wave elevation and roll angle exist in the most probable scenario using $u_{i}, \bar{u}_{i}$ as optimization parameters whereas the properties of PSWF implies nearly no wave and response here.

The reliability index $\beta$

$$
\beta=\sqrt{\sum_{i=1}^{M}\left(u_{i}^{* 2}+\bar{u}_{i}^{* 2}\right)}=\sqrt{\sum_{n=0}^{N_{e}} z_{n}^{* 2}}
$$

is a strong statistical measure as the probability of exceeding a given roll angle $\theta_{0}$ in a stationary stochastic sea during at time $\tau$ (with a mean zero up-crossing rate $v_{0}$ ) can be estimated by, e.g. Choi et al. (2017)

$$
P\left[\max _{\tau} \theta(t)>\theta_{0}\right]=1-\exp \left(-v_{0} \tau \exp \left(-0.5 \beta\left(\theta_{0}\right)^{2}\right)\right)
$$




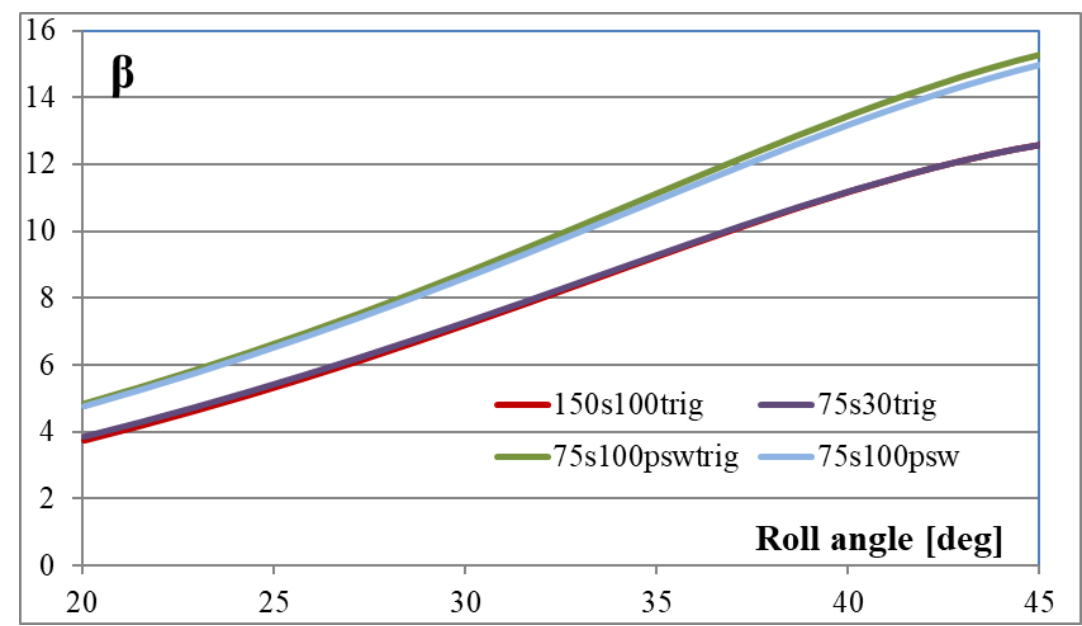

Fig. 5. Reliability index $\beta$ as function of roll angle

The FORM procedure provides as a main result the reliability index and it has been found in numerous studies, e.g. Choi et al. (2017), that the FORM results often are very close to results from Monte Carlo simulations when dealing with wave responses. The reliability index from the present study is shown in Fig. 5. A significant difference between the results using $u_{i}, \bar{u}_{i}$ and $z_{i}$ as optimization parameters are clearly seen. The reliability index is much larger for the PSWF wave representation and will thus give a very un-conservative estimate of the probability of exceeding a given roll angle. The reason for the large difference must be the nearly zero wave elevation between -75 sec $\leq t<-25$ sec using PSWF. This property with PSWF removes a large part of probable wave scenario that could give raise to roll angles exceeding $\theta_{0}$ and therefore the PSWF basic functions cannot be considered useful in the present FORM context.

\section{Conclusions}

The paper consider the use of prolate spheroidal wave functions (PSWF) as basic orthogonal functions in the wave elevation description in the time domain first order reliability method applied to wave responses in stationary sea states. Intact stability of a ship in beam sea is considered as an example and the results for the most probable response and the associated statistics are evaluated as compared to the usual procedure using trigonometric functions. The finding is, unfortunately, that the procedure is not useful due to the rapid decay of the PSWF outside a zone close to the target response. Thereby, wave scenarios, which could lead to exceedance of the target response, are greatly reduced implying a very un-conservative estimate of the probability of exceedance. If the time zone is increased to the size of the hydrodynamic memory the problem is probably solved, but in that case the number of PSWF needed will be comparable with the number of trigonometric functions normally used in FORM and thereby no 
notably gain using PSWF will be achieved. Furthermore, for $c>20$, the present very efficient procedure, Eq. (2), for construction of PSWF breaks down due to too many eigenvalues close to unity (within 12 digits) and a more elaborate procedure based on expansion in Legendre polynomial, e.g. Xiao et al. (2001), must then be applied.

The problem is in some way general as can be seen from the autocorrelation function for a constant spectrum with lower bound zero and upper bound $\Omega$ : $R(t) / R(0)=\sin (\Omega t) / \Omega t$. If the memory time $T$ is defined as a reduction of $R(t)$ by a number $C$, then $\Omega T=C$. Thus the reduction $C$ resembles the Slepian frequency $C$, Eq. (5), and shows that a lower upper bound frequency $\Omega$ (i.e. a more narrow-banded process) is counteracted by a longer memory $T$. The memory time relates to the wave elevation process and if the response process has a shorter memory time the procedure might work. However, that is not usually the case as the filtering of the wave spectrum with the response amplitude operator normally makes the response spectrum more narrow-banded implying a longer memory time.

\section{References}

Choi J-H, Jensen JJ, Kristensen HO, Nielsen UD, Erichsen, H: Intact stability analysis of dead ship conditions using FORM. JOURNAL OF SHIP RESEARCH 61(3), 167-176, (2017)

Jensen JJ: Stochastic procedures for extreme wave load predictions - Wave bending moment in ships. MARINE STRUCTURES 22(2), 194-208 (2009)

Jensen JJ: Conditional stochastic processes applied to wave load predictions. Weinblum Memorial Lecture, 2014. J. SHIP RESEARCH 59(1), 1-10, (2015)

Khare, K, George, N: Sampling theory approach to prolate spheriodal wavefunctions. J. PHYSIC. A: MATH. Gen. 36, 10011-10021 (2003)

Sclavounos, PD: Karhunen-Loeve representation of stochastic ocean waves. Proc. R. Soc. A 468, 2574-2594 (2012)

Xiao, H, Rokhlin V, Yarvin, N: Prolate spheroidal wavefunctions, quadrature and interpolation. Inverse Problems 17, 805-838 (2001) 